\title{
Influence of chlorinated water on the development of allergic diseases - An overview
}

\author{
Alina Kanikowska ${ }^{1, A-F}$, Katarzyna Napiórkowska-Baran²,B,D, Małgorzata Graczyk ${ }^{2, B, D}$, \\ Marcin A. Kucharski ${ }^{1, B, D, F}$ \\ ${ }^{1}$ Department of Gastroenterology, Metabolic and Internal Diseases and Dietetics, Poznań, Poland \\ ${ }^{2}$ Department of Allergology, Clinical Immunology and Internal Diseases, Bydgoszcz, Poland \\ A - Research concept and design, B - Collection and/or assembly of data, C - Data analysis and interpretation, \\ $D$ - Writing the article, E-Critical revision of the article, F- Final approval of the article
}

Kanikowska A, Napiórkowska-Baran K, Graczyk M, Kucharski MA. Influence of chlorinated water on the development of allergic diseases - An overview. Ann Agric Environ Med. 2018; 25(4): 651-655. doi: 10.26444/aaem/79810

\begin{abstract}
Indoor swimming pools can be used all year round and serve for one of the most popular sport pursued for recreation The positive effect of swimming arises in particular from the involvement of all the muscles of the body, decreasing the burden on the joints, as well as functional improvement of both the lungs and heart. Chlorine is typically used to disinfect swimming pool water and as a result the changes that take place lead to the formation of by-products, such as monochloramines $\left(\mathrm{NH}_{2} \mathrm{Cl}\right)$, dichloramines $\left(\mathrm{NH}_{2} \mathrm{Cl}_{2}\right)$ i trichloramines $\left(\mathrm{NH}_{2} \mathrm{Cl}_{3}\right)$, trihalogenometans (THM) or haloacetic acid (HAA). The highest concentration of these substances is just above the water surface and they may cause irritation of skin, eyes and mucosa of the respiratory tract. The toxic effect of high chlorine concentration and its side-products on the respiratory system is known, but the effect of low concentrations of these compounds is still not fully determined. Recent studies suggest that development of allergic diseases among swimmers may be increased by epithelial disorders driven by airway barrier dysfunction caused by chlorine irritation. Swimming in chlorinated water may be linked to symptoms of bronchial hyperreactivity, asthma and rhinitis especially in children, elite swimmers and employees of indoor swimming pools. Hypersensivity pneumonitis related to the use of swimming pools may manifest as a swimming pool or sauna user lung, most commonly caused by water polluting pathogens. The article summarizes recent data concerning the influence of chlorinated water on the development of allergic diseases.
\end{abstract}

\section{Key words}

asthma, allergic rhinitis, allergic diseases, swimming pool, chlorinated water

\section{INTRODUCTION}

Swimming is a sports activity linked to aerobic effort, recommended for both children and older people, with different illnesses, especially with increased body mass, rheumatologic, neurological diseases or cardiovascular disorders. The positive effect of activity in water arises in particular from the involvement of all the muscles of the body, decreased burden on the joints, as well as functional improvement of both the lungs and heart. Because of the accessibility of indoor swimming pools, this sport can be performed all year round and is one of the most popular sports pursued for recreation [1].

The presented study summarizes the current state of research on the influence of chlorinated water on the development of allergic diseases, with the focus on the impact of chlorinated water in swimming pools on the development of bronchial hyperreactivity, asthma and rhinitis. Between 1 January 2007 - 31 March 2017, the Internet websites Pubmed, ClinicalKey and Google Scholar were searched, using the keywords: chlorinated water, swimming pool, allergic diseases, asthma, rhinitis. Only English language articles were selected.

Regular physical activity in people suffering from bronchial asthma is significant in relation to improvement of general

Address for correspondence: Alina Kanikowska, Department of Gastroenterology, Metabolic and Internal Diseases and Dietetics, Przybyszewskiego 49, 60-355 Poznań, Poland

E-mail: alinablaz@yahoo.com

Received: 28.05.2017; accepted: 06.11.2017; first published: 10.01.2018 physical endurance, as well as lung capacity parameters, although it is known that increased physical activity may cause exertional bronchial spasm and exacerbation of bronchial asthma $[2,3]$. During a training session, bronchial ventilation increases 20-30 times which may originate a change in the breathing pattern from the nasal to mixed. Hyperventilation and cold air may induce bronchial spasm through water loss and increased osmolarity of bronchial tissues which, in turn, causes the release from cells of inflammatory mediators, such as histamine, prostaglandin and leukotrienes. Furthermore, increased bronchial ventilation fosters penetration of the air pollutants, allergens as well as other irritating factors throughout the respiratory tract mucous membrane [4]. Damage to the epithelium can take place through this mechanism and lead to chronic bronchial inflammation with possible remodeling of the pulmonary tract. This concerns many branches of sports. Most commonly this phenomenon is described among skiers, but is also observed in swimmers, although in reference to this group of sportsmen, the moist environment and higher air temperature partially limit this reaction [5].

Swimming is associated with smaller intensity of postexertional bronchial spasm, compared with running or riding a bicycle with the same intensity. Average air humidity in an indoor swimming pool is around 60\%, and the average air temperature $27^{\circ} \mathrm{C}$. The moist and warm air breathed by the people using the swimming pool causes less frequent occurrence of asthma exacerbation or bronchial spasm related to exertion. Furthermore, the influence of water 
pressure on the walls of the chest assists exhalation, as well as the retention of $\mathrm{CO}_{2}$ attributed to secondary hypoventilation and controlled breathing during swimming, which assists in bronchial relaxation [1].

Despite the positive aspects of swimming, the use of indoor swimming pools with chlorinated water may lead to the development of bronchial hyperreactivity, even in a healthy person. Recently, more attention is being paid to the potential role of chlorine compounds in the damage of the respiratory tract mucous membrane and the skin [6]. People spending a long time in contact with these substances - lifeguards, cleaning personnel, as well as professional swimmers, are those most susceptible to the negative action of the chloride compounds [7]. However, there is a possibility of toxic action of those compounds on people swimming recreationally, small children in particular $[8,9]$.

Water chlorination. Chlorination with the use of sodium hypochlorite or gaseous chlorine is the most commonly used method to disinfect swimming pool water all worldwide. Contact of chlorine with organic compounds added to the water, together with the submergence of a swimmer's body, and originating from the epidermis, urine, sweat, remnants of cosmetics and unwashed impurities, chloramines and other chlorinated side compounds are formed. These are, among others, monochloramines $\left(\mathrm{NH}_{2} \mathrm{Cl}\right)$, dichloramines $\left(\mathrm{NH}_{2} \mathrm{Cl}_{2}\right)$ i trichloramines $\left(\mathrm{NH}_{2} \mathrm{Cl}_{3}\right)$, as well as organohalogen compounds, such as trihalogenometans (THM) or haloacetic acid (HAA). Since 1953, chloramines have been considered as the cause of skin, eye and respiratory tract irritation [10].

Trichloramine is included among the most toxic of substances. Trichloramine is a toxic compound, easily volatile, with a characteristic smell, which hovers over indoor swimming pool buildings, and which in higher concentrations causes acute irritation to the respiratory tract mucous membrane [11]. Seys et al. described a situation in which, as a result of an incidental increase of trichloramine concentration in the air, people being present in indoor swimming pools complained of cough, dyspnea, eye lacrymation and nasal congestion. In 22 out of 26 people, bronchial hyperactivity was discovered during a provocation trial with histamine [12].

In turn, trihalogenomethans, among which the most wellknown compound is chloroform, in high concentrations cause depression of the central nervous system and damage to the heart muscle. In animals, chronic exposure to trihalogenomethans cause the development of kidney neoplasms. These compounds are also connected with higher risk of occurrence of bladder neoplasms in humans, and possible impairment of the reproductive system $[10,13,14]$.

The toxic effect of high chlorine concentration and its side products on the respiratory system is known, but the effect of low concentrations of these compounds is still not fully determined. The highest concentration of these substances is just above the water surface. In regular swimmers, a shift from nasal to mouth breathing occurs at ventilation $22 \mathrm{l} / \mathrm{min}$, resulting in less efficient clearance of chlorinated by-products and higher concentration of these substances in the lungs [15].

Because of the observed fact of the more frequent occurrence of allergic disorders, including bronchial asthma, in professional swimmers and in people with close contact to chlorinated water, research was conducted on the effect of chronic exposure to low concentrations of chlorine compounds on the human body. To-date, a dozen or so research papers have been published evaluating the effect of chlorinated water on the respiratory system $[16,17,18]$.

Bronchial hyperactivity, bronchial asthma. Some research does not confirm the negative influence of chlorine compounds on the development of bronchial asthma and other allergic disorders. There is also the possibility of reverse causation explaining this effect, because swimming is also often recommended for people with asthma as a tolerated sport that improves lung function.

British research from the neonatal period was carried out in a group of 5,738 children to assess increased risk of allergic disorders as a result of regular attendance to the swimming pool, performed with spirometry assessment and respiratory skin prick tests in $7^{\text {th }}$ and $10^{\text {th }}$ years of life. The research, however, did not confirm such a dependence, although it did show improvement in lung capacity and decrease in the incidence of occurrence of asthma in children in whom obstruction of the respiratory tract had been previously diagnosed. Swimming pool attendance was not associated significantly with bronchial hyperreactivity [16]. Another meta-analysis in 5,851 subjects did not show a significant difference in asthma development between children attending swimming pools and controls [19]. In a Dutch study assessing 2,359 children aged $6-13$ years, statistical analysis showed that swimming pool attendance was not associated with respiratory symptoms in the studied group; however, frequent baby swimming was associated with sensitisation to house dust mites [20].

Font-Ribera et al., analysed questionnaires completed by the parents of 2,758 children aged 6-12 years from 18 primary schools in Barcelona Province, Spain, and the conclusion of this cross-sectional study was that regular indoor swimming before 2 years of age, and current swimming for the last 12 months, was not related to asthma, wheezing or eczema [21].

However, increasingly more studies show that chlorine compounds may be responsible for the development of bronchial hyperreactivity, promote the development of allergies to aeroallergens, and induce mucosal membrane inflammation of the nose and eyes [5, 22, 23]. Discrepancies in the results of these studies may arise from the different norms for chlorine compounds used for water disinfection at indoor pools in different countries, as well as different age groups of studied populations.

It was observed that prolonged presence in the environment containing chlorinated compounds, such as in the case of lifeguards or maintenance workers at the swimming pool, was linked with significantly increased frequency of sinusitis, throat inflammation and chronic cough, compared with other personnel working at a distance from the chlorinated water and therefore with less exposure to irritating effect of these compounds $[24,25,26]$. An Iranian study among 38 male lifeguards and 38 controls showed that lifeguards more often had respiratory symptoms, such as bronchial hyperreactivity, cough and sputum production, especially during the work period, compared to personnel not working in a chlorinated environment [27].

In the study by Bougault et al., in bronchial mucosal membrane biopsies taken from 23 professional swimmers and compared with the control group composed of 10 healthy people and 10 people with bronchial asthma, a larger number 
of eosinophils, mast cells as well as goblet cells and mucin expression, were observed. These differences were not observed with regard to bronchial hyperactivity studied in an induced spirometry trial with metacholine between the studied groups. Increased mucin production in the mucous membrane of the swimmers may have been a reaction preventing dehydration of the mucosal membranes of the respiratory tract, although it may also have been a defence reaction to the irritating chlorine compounds [28].

Research by Voisin et al., encompassing 196 children at kindergarten age showed that regular swimming in a pool with chlorinated water before the third year of life was linked to the occurrence at the age of 5-7 years of higher concentrations of sIgE for house dust mites in the serum, and higher nitrous oxide concentrations in exhaled air, which is a risk factor for the development of bronchial asthma and rhinitis. Such dependencies were not observed in reference to sIgE for cat and plant pollens [29].

In a prospective study involving a 6-year period of observation, it was confirmed that newborns who did not regularly attend a swimming pool in their first year of life, less frequently suffered from ear and respiratory tract infections, and at the age of 6 years less frequently suffered from bronchial asthma. However, according to the researcher, this effect could also have had causes other than exposure to chlorine compounds. No association was found between swimming in chlorinated water during the neonatal period and increased frequency of occurrence of atopic dermatitis or pollinosis during the 6-year observation [30].

In a large Swedish population study involving 1,652 children aged 11-12 years, it was ascertained that the children visiting a swimming pool more than once a week more often suffered from bronchial asthma than children swimming less frequently. This risk was higher only in children allergic to aeroallergen, which was confirmed by skin prick tests. No association was found, however, between swimming in chlorinated water and increase in the risk of development of atopic dermatitis or rhinitis [31]

Another study by Bernard et al. included 847 secondary school adolescents who attended outdoor chlorinated pools. The total and aeroallergen-specific IgE concentrations in serum and the concentration of nitric oxide in exhaled air were measured. It was observed that adolescents who had regularly attended an outdoor swimming pool before the age of 7 years had a higher frequency of sensitizations to aeroallergens particularly to cat or house dust mite allergens [32]. Even in adult recreational swimmers a higher frequency of new onset asthma was observed in a study of 1,136 adults, and development of the disease was associated with the cumulative lifetime hours spent in indoor swimming pools [33].

In the study by Carbonelle et al., after a one hour stay in an environment with chlorinated compounds, temporary damage to the bronchial epithelium was observed in children and adults swimming recreationally, as well as people passively inhaling air containing chlorine compounds. Such recurring damage is most probably associated with oxidational stress, and causes an inflammatory state that subsequently leads to the development of allergy to aeroallergens. Swimming in open swimming pools with chlorinated water was also associated with more frequent occurrences of asthma, aeroallergy to house dust mites, as well as increased concentration of nitrous oxide in exhaled air [34].
The effect of chlorinated water on the induction of bronchial hyperactivity seems to be underestimated. Frequently, the symptoms related to the respiratory tract that occur after a swimming session are not reported, and they are treated as symptoms typical for intensive exercise, and therefore downplayed.

In most of the swimmers, cessation of professional swimming reduces bronchial hyperactivity; however, it is not known if this is fully reversible and in what time period the decrease takes place [1].

Extrinsic allergic alveolar inflammation. Hypersensivity pneumonitis related to the use of swimming pools may manifest as swimming pool or sauna user lung, most commonly caused by Mycobacterium avium intracellulare complex, as well as Aureobasidium sp., water polluting pathogens. Cases have also been described of allergic pneumonitis in the form of a reaction to Candida albicans and Aspergillus fumigatus infections. Typical symptoms of hypersensivity pneumonitis are the occurrence about 4-8 hours after the use of a swimming pool, of fever, muscle pains, headache, chills, dry cough and dyspnea. In physical examination, typically tachycardia is present and crackles are heard on auscultation at the base of the lungs [35].

Allergic and non-allergic rhinitis. Swimming in chlorinated water also causes the occurrence of symptoms related to the nasal mucus membrane. The most common inflammation is neutrophilic inflammation of the nasal mucous membrane, resulting from the irritating effect of chlorine compounds present in the water and in the air. The use of a nose clip while swimming significantly decreases these symptoms.

In a study evaluating the effect of intensive swimming on the occurrence or exacerbation of rhinitis, in most of the studied swimmers such a dependency was confirmed. Furthermore, it was determined that rhinitis was mostly the result of the irritating effect of chlorinated water, and was not caused by allergic rhinitis [36]. Similarly, in the Alvesa et al. study, it was observed that swimmers, contrary to runners after physical exertion, experienced exacerbation and disturbance in the nasal function which was not dependent on the susceptibility to allergy, but which was mostly linked to the draining of secretions on the posterior aspect of the throat, due to the irritation by chlorine and water [37]. In turn, a study by Geraldi et al. among a group of 54 swimmers with symptoms of rhinitis, showed that $44 \%$ of those studied had eosinophilic allergic rhinitis, and in $34 \%$ of them neutrophilic inflammation was diagnosed. The use of a nose clip decreased the symptoms of rhinitis only in the group with neutrophilic inflammation [38].

Rhinitis may have a negative effect on the sport results of swimmers. In one study, the time differences covering a certain distance between swimmers with and without rhinitis was within the range for obtaining a gold medal and not winning any medals at all. This effect was not observed in the context of bronchial asthma; swimmers with bronchial asthma had similar results, and some of them obtained even better times than swimmers without bronchial asthma [39].

The effect of long exposure to chlorine by-products was evaluated in a Turkish study which revealed a higher percentage of rhinitis and number of eosinophils in nasal cytology obtained from swimming pool workers than controls [40]. 


\section{CONCLUSIONS}

In conclusion, it seems that children, elite swimmers and employees of indoor swimming pools are more prone to developing respiratory conditions, such as bronchial hyperreactivity, asthma and rhinitis, due to their increased exposure to chlorine [41].

There are no population studies comparing the effect of swimming pool use in which alternative water disinfectants are used: ozone, ultraviolet radiation, bromine and salt, as well as silver and copper ions. The optimal microbiological and chemical method is the ozonation of water; however, because of the higher cost, this method is rarely used and is also not totally devoid of toxic byproducts. In the case of use of swimming pools with chlorinated water, air circulation is crucial because it dissipates the toxic compounds suspended in air. The use of the smallest effective quantities of chlorine disinfectant, careful body washing by people using the pool before swimming, and the use of swimming caps in order to limit the delivery of organic compounds into the pool, are also important [42].

\section{REFERENCES}

1. Bougalt V, Boulet LP. Airways Disorders and the swimming pool. Immunol Allergy Clin N Am. 2013; 33: 395-408.

2. Bougault V, Turmel J, Levesque B, Boulet LP. The respiratory health of swimmers. Sports Med. 2009; 39(4): 295-312.

3. Bemanian MH, Shirkhoda S, Nakhjavani M, Mozafari H. Effect of swimming on peak expiratory flow rate of atopic children. Iran J Allergy Asthma Immunol. 2009; 8(2): 121-3.

4. Bernard A, Nickmilder M, Dumont X. Chlorinated pool attendance, airway epithelium defects and the risks of allergic diseases in adolescents: Interrelationships revealed by circulating biomarkers. Environ Res. 2015;140: 119-26.

5. Levai IK, Hull JH, Loosemore M., Greenwell J, Whyte G, Dickinson JW. Environmental influence on the prevalence and pattern of airway dysfunction in elite athletes. Respirology. 2016; 21(8): 1391-1396.

6. Chowdhury S, Alhooshani K, Karanfil T. Disinfection byproducts in swimming pool: Occurrences, implications and future needs. Water Res. 2014; 54: 68-109.

7. Mountjoy M, Fitch K, Boulet LP, Bougault V, van Mechelen W, Verhagen E. Prevalence and characteristics of asthma in the aquatic disciplines. J Allergy Clin Immunol. 2015; 136(3): 588-94.

8. Kim S-H, Park D-E, Lee H-S, Kang H-R, Cho S-H. Chronic low dose chlorine exposure aggravates allergic inflammation and airway hyperresponsiveness and activates inflammasome pathway. PloS One. 2014; 9(9): e106861.

9. Bernard A. Asthma and swimming: weighing the benefits and the risks. J Pediatr. 2010; 86(5): 351-352.

10. Villanueva CM, Cordier S, Font-Ribera L, Salas LA, Levallois P. Overview of disinfection by-products and associated health effects. Curr Environ Health Rep. 2015; 2(1): 107-15.

11. White CW, Martin JG. Chlorine gas inhalation: human clinical evidence of toxicity and experience in animal models. Proc Am Thorac Soc. 2010; 7(4): 257-63.

12. Seys SF, Feyen S, Keirsbilck S. An outbreak of swimming-pool related respiratory symptoms: An elusive source of trichloramine in a municipal indoor swimming pool. Environ Health. 2015; 218: 386-391.

13. Villaneuva CM, Cantor KP, Grimalt JO, Malats N, Silverman D, Tardon A, et al. Bladder cancer and exposure to water disinfection by-products through ingestion, bathing and swimming in pools. Am J Epidemiol. 2007; 165(2): 148-156.

14. Florentin A, Hautemaniere A, Hertemn P. Health effects of disinfection by-products in chlorinated swimming pools. International J Hyg Environ Health. 2011; 214: 461-469.

15. Lomax M. Airway dysfunction in elite swimmers: prevalence, impact, and challenges. Open Access J Sports Med. 2016;7: 55-63.

16. Font-Ribera L, Villanueva CM, Nieuwenhuijsen MJ, Zock JP, Kogevinas M, Henderson J. Swimming pool attendance, asthma, allergies, and lung function in the Avon Longitudinal Study of Parents and Children cohort. Am J Respir Crit Care Med. 2011; 183(5): 582-8.

17. Bernard A, Carbonnelle S, de Burbure C, Michel O, Nickmilder M. Chlorinated pool attendance, atopy, and the risk of asthma during childhood. Environ Health Perspect. 2006; 114: 1567-73.

18. Font-Ribera L, Kogevinas M, Zock JP, Nieuwenhuijsen MJ, Heederik D, Villanueva CM. Swimming pool attendance and risk of asthma and allergic symptoms in children. Eur Respir J. 2009; 34 : 1304-10.

19. Valeriani F, Protano C, Vitali M, Romano Spica V. Swimming attendance during childhood and development of asthma: Meta-analysis. Pediatr Int. 2016; 59(5): 614-621.

20. Jacobs JH, Fuertes E, Krop EJ, Spithoven J, Tromp P, Heederik DJ. Swimming pool attendance and respiratory symptoms and allergies among Dutch children. Occup Environ Med. 2012; 69(11): 823-30.

21. Font-Ribera L, Villanueva CM, Gràcia-Lavedan E, Borràs-Santos A, Kogevinas M, Zock JP. Indoor swimming pool attendance and respiratory and dermal health in schoolchildren-HITEA Catalonia. Respir Med. 2014; 108(7): 1056-9.

22. Martin N, Lindley MR, Hargadon B, Monteiro WR, Pavord ID. Airway dysfunction and inflammation in pool- and non-pool-based elite athletes. Med Sci Sports Exerc. 2012; 44(8): 1433-9.

23. Romberg K, Tufvesson E, Bjermer L. Asthma is more prevalent in elite swimming adolescents despite better mental and physical health. Scand J Med Sci Sports. 2012; 22(3): 362-71.

24. Jacobs JH, Spaan S, van Rooy GB, Meliefste C, Zaat VA, Rooyackers JM, et al. Exposure to chloramine and respiratory symptoms in indoor swimming pool workers Eur Resp J. 2007; 29 (4): 690-698.

25. Rosenman KD, Millerick-May M, Reilly MJ, Flattery MJ, Weinberg J, Harrison J et al. Swimming facilities and work-related asthma. J Asthma. 2015; 52(1): 52-8.

26. Nordberg GF, Lundstrom NG, Forsberg B, Hagenbjork-Gustafsson A, Lagerkvist BJ, Nilsson J, et al. Lung function in volunteers before and after exposure to trichloramine in indoor pool environments and asthma in a cohort of pool workers. BMJ Open. 2012; 2(5): e000973.

27. Boskabady MH, Esmaeilizadeh M, Boskabady M. The effect of exposure to chlorine on pulmonary function tests and respiratory and allergic symptoms in Iranian lifeguards. Toxicol Ind Health. 2014; 30(3): $218-24$

28. Bougault V, Loubaki L, Joubert I, Turmel J, Couture C, Laviolette M, et al. Airway remodeling and inflammation in competitive swimmers training in indoor chlorinated swimming pools. J Allergy Clin Immunol. 2012; 129: 351-8.

29. Voisin C, Sardella A, Bernard A. Risks of new -onset allergic sensitization and airway inflammation after early age swimming in chlorinated pools. Int J Hyg Environ Health. 2014; 217: 38-45.

30. Schoefer Y, Zutavern A, Brockow I, Schäfer T, Krämer U, Schaaf B, et al. Health risks of early swimming pool attendance? Int J Hyg Environ Health. 2011; 304: 367-373.

31. Andersson M, Hedman L, Nordberg G, Forsberg B, Eriksson K, Rönmark E. Swimming pool attendance is related to asthma among atopic school children: a population- based study. Environ Health. 2015; 14: 37.

32. Bernard A, Nickmilder M, Voisin C. Outdoor swimming pools and the risks of asthma and allergies during adolescence. Eur Respir J. 2008; 32: 979-988.

33. Ferrari M, Schenk K, Mantovani W, Papadopoulou C, Posenato C, Ferrari $\mathrm{P}$, et al. Attendance at chlorinated indoor pools and risk of asthma in adult recreational swimmers. J Sci Med Sport. 2011; 14(3): 184-9.

34. Carbonelle S, Francaux M, Doyle I, Dumont X, de Burbure C, Morel $\mathrm{G}$, et al. Changes in serum pneumoproteins caused by short-term exposures to nitrogen trichloride in indoor chlorinated swimming pools. Biomarkers. 2002; 7: 464-78.

35. Sood A, Sreedhar R, Kulkarni P, Nawoor AR. Hypersensitivity pneumonitis-like granulomatous lung disease with nontuberculous mycobacteria from exposure to hot water aerosols. Environ Health Perspect. 2007; 115(2): 262-6.

36. Bougault V, Turmel J, Boulet LP. Effect of intense swimming training on rhinitis in high-level competitive swimmers. Clin Exp Allergy. 2010; 40(8): 1238-46.

37. Alves A, Martins C, Delgado L, Fonseca J, Moreira A. Exercise-induced rhinitis in competitive swimmers. Am J Rhinol Allergy. 2010; 24(5): e114-7.

38. Gelardi M, Ventura MT, Fiorella F, Fiorella ML, Russo C, Candreva $\mathrm{T}$, et al. Allergic and nonallergic rhinitis in swimmers: clinical and cytological aspects. Br J Sports Med. 2012; 46(1): 54-8. 
39. Stadelmann K, Stensrud T, Carlsen K-H. Respiratory symptomoms and bronchial responsiveness in competitive swimmers. Med Sci Sports Exerc. 2011; 43: 375-81.

40. Erkul E, Yaz A, Cingı C, İnançli HM, San T, Bal C. Effects of indoor swimming pools on the nasal cytology of pool workers. J Laryngol Otol. 2014; 128(5): 442-6.

41. Angione S, McClenaghan H, LaPlante A. A Review of Chlorine in Indoor Swimming Pools and its Increased Risk of Adverse Health
Effects. Revue interdisciplinaire des sciences de la santé- Int J Health Sci. 2011; 2(1): 44-51.

42. Weisel CP, Richardson SD, Nemery B, Aggazzotti G, Baraldi E, Blatchley ER 3rd, et al. Childhood asthma and environmental exposures at swimming pools: state of the science and research recommendations. Environ Health Perspect. 2009; 117(4): 500-7. 